\title{
THE PERIOD DISTRIBUTION OF SUPERSOFT SOURCES
}

\author{
P. KAHABKA
}

Astronomical Institute "Anton Pannekoek" and Center for

High Energy Astrophysics, University of Amsterdam, Kruislaan 403, 1098 SJ Amsterdam, The Netherlands

\begin{abstract}
The observed and predicted orbital period distributions of supersoft sources are in good agreement.
\end{abstract}

\section{Results and conclusions}

Presently for 8 of 10 optically identified supersoft systems binary orbital periods have been determined (Kahabka \& Trümper 1995, Kahabka 1995a). These systems can be classified into 3 groups: classical novae (with two members), unstable mass transfer (canonical) systems (with six members) and symbiotic novae (with one member). We consider only the period distribution of the canonical systems. The period distribution of this class extends from $4.1 \mathrm{~h}$ to $3.5 \mathrm{~d}$. Rappaport, di Stephano \& Smith (1994) simulated the orbital period distribution of the galactic supersoft systems. In model 7 , in which the time evolution of the binary has explicitly been considered, a

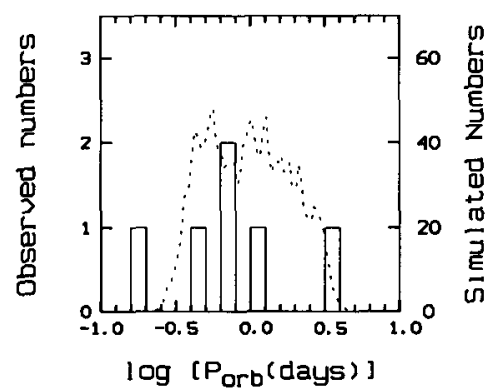

Figure 1. Orbital period distribution of supersoft sources. Only the distribution of the canonical systems (evolved main-sequence or post main-sequence star binaries) is shown.

421

A. Evans and J. H. Wood (eds.), Cataclysmic Variables and Related Objects, 421-422.

(C) 1996 Kluwer Academic Publishers. Printed in the Netherlands. 
TABLE 1. Supersoft systems for which orbital periods have been determined

\begin{tabular}{llll}
\hline Name & $\begin{array}{l}\mathrm{P}_{\text {orb }} \\
{[\mathrm{d}]}\end{array}$ & $\log \left(\mathrm{P}_{\text {orb }}\right)$ & remarks \\
\hline Nova Mus 1983 (GQ Mus) & 0.0593 & -1.22 & classical nova \\
Nova V1974 Cyg (Nova Cyg 1992) & 0.0812 & -1.09 & classical nova \\
1E0035.4-7230 & 0.172 & -0.764 & variable \\
CAL 87 & 0.442 & -0.353 & persistent \\
RX J0019.8+2156 & 0.660 & -0.180 & recurrent \\
RX J0513.9-6951 & 0.76 & -0.119 & recurrent \\
CAL 83 & 1.04 & +0.0170 & persistent \\
RX J0925.7-4758 & 3.4 & +0.544 & persistent \\
AG Dra & 554 & +2.74 & symbiotic nova \\
\hline
\end{tabular}

distribution containing $\sim 1800$ members and extending from $\sim 4.3 \mathrm{~h}$ to $\sim 4 \mathrm{~d}$ is predicted. This can be considered to be a good overall agreement with the observed distribution and is in strong support for the steady-state nuclear burning model being applicable. Only the $4.1 \mathrm{~h}$ period, found in the (only) SMC binary system 1E0035.4-7230 may be of concern. This could be in favor for a recurrently nuclear burning and lower mass white dwarf (in order to keep it sufficiently long burning in agreement with the observations). Recurrent supersoft sources ( $\mathrm{cf}$. Kahabka 1995b) have been found to have slightly lower mass accretion rates $\left(\sim 810^{-8} \ldots 410^{-7} \mathrm{M}_{\odot} \mathrm{yr}^{-1}\right)$ and higher white dwarf masses $\left(1.0 \ldots 1.4 \mathrm{M}_{\odot}\right)$ compared to steady-state nuclear burning systems (cf. van den Heuvel et al. 1992). The observed systems most probably have already accumulated a significant amount of accreted hydrogen within evolutionary time-scales and gained in mass considerably. This may affect their further evolution. For a discussion of the period distribution of the other classes of supersoft X-ray binaries (symbiotic, CV-like systems, etc.) I refer to the work of Yungelson et al. (1996).

\section{References}

Kahabka, P., Trümper J., 1995, in "IAU Symposium 165, Compact Stars in Binaries", $\mathrm{p} 425$

Kahabka, P., 1995a, in "Cataclysmic Variables", eds A. Bianchini et al., Kluwer, p435

Kahabka, P., 1995b, A\&A, 304, 227

Rappaport, S., di Stephano, R., Smith, J.D., 1994, Ap. J., 270, L9

van den Heuvel, E.P.J., Bhattacharya, D., Nomoto, K., et al., 1992, A\&A, 262, 97

Yungelson, L., Livio M., Tutukov A., Kenyon S.J., 1996, Ap. J., submitted 